diagnosed heart condition chronologically connected with the gym activities, made us suspect of abuse of anabolic steroids as the cause of hypertrophic cardiomyopathy. A gradual decrease of the septal thickness later may be connected to the abstinence from steroids after the incident, although it also may be attributed to ischemic myocardial necrosis.

Synthetic cannabinoids, usually sold as cheap air fresheners, are known to cause cardiac infarction and arrest. They are difficult to prove by laboratory tests. Sources other than the patient and his parents strongly suggested that the patient took those substances just before the cardiac arrest. We presume that there is a significantly greater risk of a life-threatening arrhythmia and/or cardiac arrest in the case of hypertrophic cardiomyopathy if the patient abuses synthetic cannabinoids.

\section{RARE CASE OF EXTENSIVE DEEP VEIN THROMBOSIS AND GIANT THIGH ABSCESS DUE TO STREPTOCOCCUS PYOGENES SEPSIS}

G Plosnic*, T Matic, F Rubic, M Cvitkovic, S Galic, S Dessardo. University Hospital Centre Zagreb

\subsection{6/archdischild-2021-europaediatrics.330}

We report a case of extensive deep venous thrombosis (DVT) and giant thigh abscess associated with Streptococcus pyogenes sepsis.

A five year old girl was referred to our PICU with an extensive DVT of the right leg confirmed by CT scan and with $48 \mathrm{~h}$ history of fever. There was no evidence of abscess or other abnormalities. Three days before admission, she fell from her bicycle and broke a tooth. The older brother had streptoccocal pharyngitis. Initial laboratory findings showed CRP $360 \mathrm{mg} / \mathrm{dL}$, PCT $78 \mathrm{ng} / \mathrm{ml}$, leucocytosis (20.100/uL), neutrophilia (banded $6 \%$ and segmented $84 \%$ ), normal haematocrit and low platelet count $(48 / \mathrm{uL})$. The remaining laboratory values, including electrolytes and coagulation tests were normal. Eventually, Streptococus pyogenes was isolated from blood culture. Subcutaneous enoxaparin was started as well as antibiotic therapy - with vancomycin, klyndamicin and meropenem. The observed progression of leg swelling raised the suspicion of a cellulits or DVT progression with developing compartment syndrome and the patient underwent surgical exploration. Apart from enlarged, reactive lymph nodes, there were no abnormalities found intraoperatively. However, after 5 days of treatment her thigh became paintfull, red and warm. Control CT scan showed a huge thigh abscess and prompt evacuation of abscess with debridement of necrotic tissue was performed. Eventually we confirmed FV Leiden mutation, as well as low protein $\mathrm{C}$ and high homocistein levels.

Infectious diseases are often accompanied by activation of coagulation.

Cytokines are believed to be important mediators in this process. A critical role for antecedent soft-tissue injury has been well established and microorganisms likely hematogenously translocated from throat to deep soft tissues (in this case by a broken tooth). Vein thrombosis often accompanies or precedes abscess formation. However, massive DVT should always raise suspicion for trombophilia.

CT and MRI scans should be interpreted with caution because S. pyogenes doesn't promote forming of gas in the tissues or form free abscesses, so radiologist's interpretations are frequently not definitive.

Prompt and aggressive surgical exploration and debridement of suspected deep-seated streptococcal infection are mandatory, as well as anticoagulant therapy. If treated properly, morbidity could be significantly reduced.

\section{THE ROLE OF AMBULANCE IN HEALTH SYSTEM}

Lilit Shahumyan*, E Harutyunyan, A Aghajanyan, A Asatryan, H Shahumyan, S Khachatryan, I Atoyan, A Sahakyan, G Manukyan, K Esoyan, S Mirabyan, A Sahakyan, M Grigoryan, B Baveyan, V Asoyan, H Apresyan. Yerevan State Medical University

\subsection{6/archdischild-2021-europaediatrics.331}

The aim of our research was to investigate the diseases of 0 18 year old patients admitted to the 'Muratsan' University hospital complex(UHC) Intensive care unit (ICU) and toxicology for the last 7 years, including cases delivered to the hospital by the ambulance service and analysis of their final diagnoses.

A retrospective study was performed using medical charts up to 7 years (2012-2018) from 'Muratsan' University hospital complex(UHC) ICU and toxicology. The overall number of patients was 5152, among them 3074 were male and 2078 were female. In this study we have included the most typical diseases (pathologies) for each particular season. This study included cases delivered to the hospital by the ambulance service and analysis of their final diagnoses.

According to the results of our study, 1695(32,8\%) patients were hospitalized with an ambulance: $164(9,7 \%)$ of them had Acute respiratory infections(ARI), 780(46\%) had Acute Intoxications(AI), which is seasonal and includes mostly carbon monoxide(CO) intoxication, $\mathrm{KMnO} 4$, flavor essence and alcohol poisoning. $375(22,1 \%)$ patients had Acute Drug Intoxications(ADI), 81(4,8\%) had complaints of allergy. 295(17,4\%) of patients had other disorders*. Patients were divided into 4 age groups $(0-1 ; 1-7 ; 7-14 ; 14-18)$, the first group-189, the second-922, the third-334 and the fourth-250. 669 (39.5\%) of cases were moderate, 968 (57.1\%)-heavy and 22 (1.3\%)extremely heavy (two of which ended in death), $36(2.1 \%)$ cases have not been reported.

*Diabetes mellitus, epilepsy, cancer, hematological disorders, etc.

We found out that the two most common diagnoses of patients who were admitted to the hospital by ambulance are $\mathrm{ADI}$ and $\mathrm{AI}$ independent of the season. The prevalence of moderate cases is also high, which may be related to the level of care provided by parents to their children. Low mortality level of extremely heavy patients who were arrived at the hospital by ambulance lets us consider about the quality of ambulance function.

\section{WITH OR WITHOUT REFERRAL?}

Hasmik Shahumyan*, E Harutyunyan, L Shahumyan, A Asatryan, A Aghajanyan, S Khachatryan, I Atoyan, A Sahakyan, G Manukyan, M Grigoryan, K Esoyan, S Mirabyan, A Sahakyan, M Grigoryan, B Baveyan, V Asoyan, H Apresyan. Yerevan State Medical University

10.1136/archdischild-2021-europaediatrics.332 
The aim of our research was to investigate the diseases of 0 18 year old patients who were admitted to the 'Muratsan' University hospital complex(UHC) Intensive care unit (ICU) and toxicology for the last 7 years, including cases that have been reported from the dispensary with or without referral.

A retrospective study was performed using medical charts up to 7 years (2012-2018) from 'Muratsan'(UHC) ICU and toxicology. The overall number of patients was 5152, among them 3074 were male and 2078 were female.

This study included cases that have been reported by the 'Muratsan'UHC ICU and toxicology from the dispensary with or without referral.

The number of patients who were referred from the polyclinics of Yerevan is 41(0.7\%). 23(56.1\%) of them had Acute respiratory infections(ARI), 3(7.3\%)-Acute Intoxications(AI), 5 (12.2\%)-Acute Drug Intoxications(ADI) and 2(4.9\%)-allergic disorders, $8(19.5 \%)$ other disorders*. The referred patients were divided into 4 age groups(0-1,1-7,7-14,14-18), the first group-10, the second-27, the third-3 and the fourth-1 case. 24\%-moderate, 64\%-severe and 11\%-extremely severe cases (the level of severity was absent in $1 \%$ of cases).

53 patients $(1 \%)$ were referred from regional polyclinics, 20 (37.7\%) of them had ARI, 13(24.5\%)-AI, 11(20.8\%)-ADI and 2(3.8\%)-allergic disorders, $7(13.2 \%)$ other disorders. The first age group included-6, the second-42, the third-5 and the fourth-0 cases. 36\%-moderate, 57\%-severe cases(the level of severity was absent in $7 \%$ of cases).

Out of 2414 patients who were admitted to hospital without referral $519(21.5 \%)$ had ARI, 859(35.6\%)-AI, 575 (23.8\%)-ADI and 87(3.6\%)-allergic disorders.

$374(15.5 \%)$-other disorders. The first age group included300 , the second-1832, the third-209 and the fourth-73 cases. $42 \%$-moderate, 54\%-severe and 2\%-extremely severe cases(the level of severity was absent in $2 \%$ of cases).

*Diabetes mellitus, epilepsy, cancer, hematological disorders, etc.

ARIs are more prevalent in patients who have been referred from polyclinics, while the patients without referral had mostly ADIs and AIs. The hospitalization without referral was rather high, which suggests that the primary chain of trust has decreased.

\section{AGE-RELATED DISORDERS IN CHILDREN}

Eline Harutyunyan*, A Aghajanyan, L Shahumyan, A Asatryan, H Shahumyan, S Khachatryan, I Atoyan, A Sahakyan, G Manukyan, K Esoyan, S Mirabyan, A Sahakyan, M Grigoryan, B Baveyan, V Asoyan, H Apresyan. Yerevan State Medical University

\subsection{6/archdischild-2021-europaediatrics.333}

The aim of our research was to investigate the diseases of 0 18 years old patients who were admitted to the 'Muratsan' University hospital complex(UHC) Intensive care unit (ICU) and toxicology for the last 7 years, their relation to the ages and the underlying reasons.

A retrospective study was performed using medical charts up to 7 years(2012-2018) from 'Muratsan' UHC ICU and toxicology. The overall number of patients was 5152, among them 3074 were male and 2078 were female.
In this study, we have separated four age groups $(0-1 ; 1-7 ; 7-$ $14 ; 14-18)$ and have included the most common disorders typical for each age group.

During this period 538 patients under 1 year old have been admitted to hospital. 201(37.4\%)-Acute Intoxications (AI) (predominantly caused by $\mathrm{KMnO} 4$ solution, carbon monoxide (CO) and flavor essence). 193(35.9\%) patients had Acute respiratory infection(ARI) and 108(20\%)-acute drug intoxications (ADI), 36(6.7\%)-other disorders*.

By observing the 3585 patients aged 1-7 years old we found out the following results: AI-1441(40.2\%) patients (acetone solution, sodium hypochlorite solution and $\mathrm{KMnO} 4$ solution), ADI-939(26.2\%), ARI-762(21.3\%), 443(12.3\%)-other disorders.

The number of 7-14 years old patients was 632, among them 316(50\%) had AI (CO, alcohol, flavor essence), 93 (14.7\%)-ADI (15\% of which were suicide attempts) and 57 (9\%)-ARI, 166(26.3\%)-other disorders.

The analysis of 361 patients aged 14-18 years old led to following results: 155(43\%)-AI (commonly with alcohol, CO, sodium hypochlorite solution), 122(33.8\%)-ADI, $75.4 \%$ of which were suicide attempts, 20(5.5\%) were bitten by animals and insects, 64(17.7\%)-other disorders.

*Diabetes mellitus, allergic disorders, cancer, hematological disorders, etc.

We found out that the most common diagnoses of the patients are ADI and AI independent of age group. This phenomenon might be associated with high curiosity of kids as well as with parental negligence. AIs in adolescents are predominantly caused by alcohol. Both ADIs and AIs in adolescents are usually due to suicide attempts.

\section{SYNCOPE IN PEDIATRIC EMERGENCY DEPARTMENT}

Nikolina Benco*, Alen Švigir, Iva Topalušić, Katarina Vulin, Zdenka Pleša Premilovac. Children's Hospital Zagreb

\subsection{6/archdischild-2021-europaediatrics.334}

The objective of this study was to evaluate the various factors related to the children who presented to the emergency department of Children's Hospital Zagreb after experiencing syncope.

Information about all visits in ER during 2018 was analyzed from a database.

Out of all 14115 visits, the syncope was present in 96 patients $(0.6 \%)$.

Their medical history, clinical characteristics, laboratory and diagnostic tests were analyzed.

The patients were mainly girls (68\%). The mean age of the patients did not differ between genders (girls $12.84 \pm 3.86$ and boys $12.71 \pm 3.54 ; \mathrm{p}=0.868$; age range $3-18 \mathrm{y}) .79 .1 \%$ arrived with an ambulance, while the rest were referred by their primary pediatrician. $57 \%$ of the patients arrived between the 08 a.m. and 4 p.m., $32 \%$ between 4 p.m. and 10 p.m., while $10 \%$ came during the night. 29\% arrived during weekend and $71 \%$ during workdays. $82 \%$ came during the school-year, thus making it obvious that syncope episodes are more often while school obligations last. 60\% experienced syncope for the first, second $(19 \%)$ and third $(11 \%)$ time. In $85 \%$ of patients the 